\title{
ON SYSTEM IDENTIFICATION AND MODEL VALIDATION VIA LINEAR PROGRAMMING
}

\author{
T.K. Gustafsson and P.M. Mäkilä \\ Åbo Akademi University, Dept of Engineering \\ $20500 \AA$ bo, FINLAND \\ pmakila@abovax.abo.fi
}

\begin{abstract}
Linear programming methods for discrete $l^{1}$ approximation are used to provide solutions to problems of approximate identification with state space models and to problems of model validation for stable uncertain systems. Choice of model structure is studied via Kolmogorov $n$-width concept and a related $n$-width concept for state space models. Several results are given for FIR, Laguerre and Kautz models concerning their approximation properties in the space of bounded-input bounded-output (BIBO) stable systems. A robust convergence result is given for a modified least sum of absolute deviations identification algorithm for BIBO stable linear discrete-time systems. A simulation example with identification of Kautz models and subsequent model validation is given.
\end{abstract}

\section{Introduction}

Recently the principle of unfalsified modelling has received increasing interest as one of the main scientific principles providing a solid paradigm of system identification for robust control design (see e.g. $[15,14,19,4])$. For various recent approaches to problems of identification for robust control design see also e.g. $[7,2,5]$.

In the present work we study decomposing the task of modelling into identification of nominal models, as best approximations within a chosen model class, followed by feasibility analysis with unmodelled dynamics. As the feasibility analysis of uncertainty models is a computationally demanding step, we are in the present work interested in formulating the identification and model validation steps in a unified manner as linear programming problems so as to facilitate realistic simulations (for testing purposes) and applications. For this reason we also use fairly general state space model structures [16] rather than just FIR models, cf. also the recent exponential time complexity re- sults in worst-case identification [3]. This allows taking into account a priori information about the system dynamics via the model structure. In the present work discrete Laguerre and Kautz models [6],[17-18] are considered in some detail. Next we give some notation.

Let $Z, R, R^{m}$ denote the integers, the reals and the set of all $m \times 1$ vectors with entries in $R$, respectively. Furthermore, let $l^{\infty}, l^{1}$ denote the spaces of (onesided) sequences $x=\left\{x_{k} \in R\right\}_{k \geq 1}$ such that $\|x\|_{\infty} \equiv$ $\sup _{k \geq 1}\left|x_{k}\right|<\infty$ and such that $\|x\|_{1} \equiv \sum_{k>1}\left|x_{k}\right|<$ $\infty$, respectively. We shall use the notation $l^{\infty}$ for the space of bounded double-sided sequences, too. Let $G: 1^{\infty} \rightarrow l^{\infty}$ denote a strictly causal linear shiftinvariant system defined as a convolution operator

$$
(G * u)(t)=\sum_{k \geq 1} g(k) u(t-k)
$$

where * denotes the convolution product, and $\{g(k) \in$ $R\}_{k \geq 1}$, is the (unit) impulse response of $G$. It is wellknown that the system $G$ is $1^{\infty}$ stable, or BIBO stable, if and only if $\|G\| \equiv \sum_{k>1}|g(k)|<\infty$. Let $B$ denote the space of strictly causal linear shift-invariant BIBO stable systems.

Let $\hat{G}$ denote the transfer function of $G \in \mathcal{B}$, defined by $\hat{G}(z)=\sum_{k \geq 1} g(k) z^{k}$, where $z$ is a complex variable. In the sequel we shall use interchangeably the notation $\hat{G}$ and $G$ for a system in $B$. Thus we define $\|\hat{G}\|$ to mean $\|G\|$, etc.

\section{Approximate Modelling with State Space Models}

Let $G_{n} \in \mathcal{B}$ be a stable $n$-dimensional state space model defined by the triple $\left(A_{n}, B_{n}, C_{n}\right)$. Keeping $A_{n}$ and $B_{n}$ fixed but varying $C_{n}$ over $R^{n}$ defines an $n$ dimensional linear subspace in $B$. The transfer function $\hat{G}_{n}(z)$ of $G_{n}$ is a rational function (in $z$ ) of degree at most $n$.

Let $G \in \mathcal{B}$. Define the approximation numbers

$a_{n}\left(G, A_{n}, B_{n}\right)=\inf _{C_{n} \in R^{n}}\left\|G-G_{n}\right\|, n=1,2, \ldots$ 
Let $G \in S \subset B$. Define the rational n-widths

$$
b_{n}(S)=\inf _{A_{n}, B_{n}} \sup _{G \in S} a_{n}\left(G, A_{n}, B_{n}\right), n=1,2, \ldots
$$

where the infimum is taken over all pairs $\left(A_{n}, B_{n}\right)$ defining stable $n$-dimensional state space models.

Introduce the Kolmogorov $n$-width of $S$ as

$$
d_{n}(S)=\inf _{\Omega_{n}} \sup _{G \in S} \inf _{F \in \Omega_{n}}\|G-F\|
$$

where the left-most infimum is taken over all $n$ dimensional linear subspaces $\Omega_{n}$ of $\mathcal{B}$. A subspace $\Omega_{n}^{*}$ achieving the infimum in (4) is an optimal subspace for $d_{n}(S)$.

Proposition 1 Let $S$ be a subset of $\mathcal{B}$. Then $b_{n}(S) \geq$ $d_{n}(S)$ for any $n$.

Theorem $1 A$ set $S$ in $B$ is relatively compact if and only if $b_{n}(S) \rightarrow 0$ when $n \rightarrow \infty$ and $S$ is bounded.

Define $A_{m}(\gamma)=\left\{G \in \mathcal{B}\|\| \hat{G}^{(m)} \| \leq \gamma\right\}, m=$ $1,2,3, \ldots$, where $\hat{G}^{(m)}$ denotes the $m$-th derivative of $\hat{G}(z)$, and $\gamma>0$. The following holds.

Theorem 2 Let $m=1,2,3, \ldots$ and $\gamma>0$. Then

$$
\begin{gathered}
b_{n}\left(A_{m}(\gamma)\right)=d_{n}\left(A_{m}(\gamma)\right)= \\
= \begin{cases}\infty, & n+1<m \\
\gamma(n+1-m) ! /(n+1) !, & n+1 \geq m .\end{cases}
\end{gathered}
$$

Furthermore, the (FIR) model set $\mathcal{P}_{n}= \begin{cases}F \in \\ \text {. }\end{cases}$ $\left.B \mid \hat{F}(z) \in \operatorname{span}\left\{z, z^{2}, \ldots, z^{n}\right\}\right\}$ is an optimal subspace for $d_{n}\left(A_{m}(\gamma)\right)$

The proofs of the above results are given in [4].

\section{Laguerre and Kautz Models}

Consider the discrete Laguerre functions $\left\{L_{k} \in \mathcal{B}\right\}_{k \geq 1}$ defined through their transfer functions as

$$
\hat{L}_{k}(z)=\frac{\sqrt{1-\alpha^{2}}}{1-\alpha z}\left(\frac{z-\alpha}{1-\alpha z}\right)^{k-1}, k=1,2, \ldots,
$$

where $-1<\alpha<1$. It is well-known that the discrete Laguerre functions $L_{k}$ form an orthonormal basis of $l^{2}$, the space of square summable real sequences.

Theorem 3 The discrete Laguerre functions are dense in $B$, i.e. the closure of the linear span of $\left\{L_{k}\right\}_{k \geq 1}$ is $B$.
Proof. We just sketch the proof. As FIR systems are dense in $\mathcal{B}$, it is enough to show that FIR systems can be approximated arbitrarily well by discrete Laguerre functions in the $\mathcal{B}$ norm. A straightforward computation shows that the discrete LaguerreFourier coefficients $c_{k}(f)$ of a Laguerre series expansion $\sum_{k \geq 1} c_{k}(f) L_{k}$ of an arbitrary FIR system $f$, decay to zero with an exponential rate when $k \rightarrow \infty$. Now, as $\left\|L_{k}\right\| \leq D k$, for some constant $D>0$, it follows that the Laguerre series of $f$ converges to $f$ in the $\mathcal{B}$ norm. This implies the result.

A discrete Kautz model with a pair of complex conjugate poles has the form

$$
\hat{K}_{n}(z)=a_{1} \hat{\psi}_{1}(z)+\ldots+a_{n} \hat{\psi}_{n}(z), a_{k} \in R,
$$

where the $\left\{\psi_{k}\right\}$ form an orthonormal basis of $l^{2}$, and each $\hat{\psi}_{k}$ has just poles at a complex conjugate pair of locations $1 / \alpha, 1 / \bar{\alpha}$, where $|\alpha|<1$. Here the functions $\hat{\psi}_{k}$ are given by

$$
\begin{aligned}
\hat{\psi}_{2 j-1}(z) & =\frac{-b \sqrt{1-c^{2}}\left(-a z+z^{2}\right)}{1+b(c-1) z-c z^{2}} S(z)^{j-1} \\
\hat{\psi}_{2 j}(z) & =\frac{\sqrt{\left(1-c^{2}\right)\left(1-b^{2}\right)} z^{2}}{1+b(c-1) z-c z^{2}} S(z)^{j-1},
\end{aligned}
$$

where $j=1,2, \ldots, a=(1+\alpha \bar{\alpha}) /(\alpha+\bar{\alpha}), b=1 / a$, and $c=-|\alpha|^{2}$. Furthermore, the function $S(z)$ is given by

$$
S(z)=\left(\frac{-c+b(c-1) z+z^{2}}{1+b(c-1) z-c z^{2}}\right) .
$$

Theorem 4 The discrete Kautz functions $\left\{\psi_{k}\right\}$ are dense in $\mathcal{B}$.

Proof. Note that as

$$
S(z)=\left(\frac{z-\alpha}{1-\bar{\alpha} z}\right)\left(\frac{z-\bar{\alpha}}{1-\alpha z}\right),
$$

the result follows from a slight modication of the argument used in the proof of the denseness of the Laguerre functions.

Discrete Laguerre and Kautz models provide two examples of state space model structures with nontrivial properties. Kautz models are especially useful for lightly damped systems [18].

\section{Identification of Nominal Models}

Let us assume that the input/output data $\{y(t), u(t)\}_{t=0}^{N-1}$ from an identification experiment is modelled as having been generated by an unknown system $G \in \mathcal{B}$ according to

$$
y(t)=(G * u)(t)+v(t),
$$


where $v(t)$ is a bounded experimental error term, and $N$ denotes the number of input/output data points available. Let $G_{n} \in \mathcal{B}$ denote a nominal model of $G$.

Whether our model priors $S_{n} \subset \mathcal{B}$ that we want the nominal model $G_{n}$ to satisfy. form a convex set or not, it is always possible to approximate a bounded set $S_{n}$ by set unions of systems of linear inequalities and linear equalities $[11,10]$.

Note that even smoothness priors of the type $\left\|\hat{G}_{n}^{(m)}\right\| \leq \gamma_{m}$ can be dealt with as linear inequalities because $\left\|\hat{G}_{n}^{(m)}\right\|=\sum_{k \geq m} k(k-1) \cdots(k-m+$ 1) $\left|C A^{k-1} B\right|$, where $\hat{G}_{n}^{(m)}(z)$ is the $m$-th derivative of $\hat{G}_{n}(z)$. Here $\gamma_{m}>0$ is a given number.

Note that also various error (noise/disturbance) priors can be expressed via linear inequalities [11]. Let $\{v(t)\}_{t=0}^{N-1}$ be a bounded noise sequence as in (12). Two examples of norm-like priors that can be handled as linear inequalities are

$$
\max _{0 \leq t \leq N-1}|v(t)| \leq \epsilon \text { and } \frac{1}{N} \sum_{t=0}^{N-1}|v(t)| \leq \zeta_{N},
$$

where $\epsilon \geq 0$ and $\zeta_{N} \geq 0$ are given numbers.

Let $G_{n}$ be a stable state space model $\left(A_{n}, B_{n}, C_{n}\right)$, where $A_{n}, B_{n}$ are given matrices and $C_{n}$ is to be in some sense optimized on the basis of input/output data. Let $\hat{x}(t)$ denote the state vector of the model at time $t$.

The identification criterion that will be of most interest here is the least sum of absolute deviations (LSAD) criterion (see e.g. [12])

$$
\inf _{C_{n} \in R^{n}} \frac{1}{N} \sum_{t=0}^{N-1}\left|y(t)-C_{n} \hat{x}(t)\right| .
$$

This criterion leads to linear programming problems when model and error priors are expressed as linear inequalities and linear equalities. It is our experience that it is possible to solve such constrained minimization problems efficiently using the method described in [1] up to at least 10000 input/output data points.

Define a black-box identification algorithm $\mathcal{A}$ as a set of mappings $\left\{\mathcal{A}_{N}: R^{N} \times R^{N} \rightarrow \mathcal{B}\right\}_{N \geq 1}$ operating on input/output data $U_{N}=\{u(t)\}_{t=0}^{N-1}, Y_{N}=$ $\{y(t)\}_{t=0}^{N-1}$ to produce models in $\mathcal{B}$ of the (unknown) system $G \in \mathcal{B}$ which generated the input/output data. We assume that the input $u(t)$ is bounded for all $t$ and possibly unknown for $t<0$. A black-box algorithm $\mathcal{A}$ is said to have the property of robust convergence in $B$ if there exists an input $\{u(t)\}_{t \geq 0}$, with $\sup _{t \geq 0}|u(t)| \leq 1$, such that

$$
\lim _{\substack{N \rightarrow \infty \\ e \rightarrow 0}} \sup _{|v(t)| \leq \epsilon, t \geq 0}\left\|G-\mathcal{A}_{N}\left(U_{N}, Y_{N}\right)\right\|=0
$$

is satisfied for any $G \in B$. It is known that the input $\{u(t)\}_{t \geq 0}$ must satisfy a strong richness property for there to exist an algorithm for which the robust convergence property is satisfied [9].

Consider modified LSAD identification using FIR models. Thus define the identification algorithm $\mathcal{L}=$ $\left\{\mathcal{L}_{N}\right\}_{N \geq 1}$ so that $\mathcal{L}_{N}\left(U_{N}, Y_{N}\right)$ is a minimizer of the modified LSAD criterion

$$
\begin{array}{r}
\inf _{F \in \mathcal{P}_{n}}\left[\frac{\lambda_{1}}{N-n} \sum_{t=n}^{N-1}|y(t)-(F * u)(t)|+\right. \\
\left.\lambda_{2} \max _{n \leq t \leq N-1}|y(t)-(F * u)(t)|\right] .
\end{array}
$$

Here $\mathcal{P}_{n}$ denotes a set of FIR models as defined in Theorem 2, and $\lambda_{1}>0, \lambda_{2}>0$ are constants such that $\lambda_{1}+\lambda_{2}=1$. Here the choice of $n$ as a function of $N$ is not arbitrary [2].

Theorem 5 The modified $L S A D$ algorithm $\mathcal{L}$ is robustly convergent in $\mathcal{B}$.

It is important to observe that the modified LSAD algorithm is nonlinear in the output data. Recent results indicate that this is essential for robust convergence [13].

\section{Model Validation for Uncertain Systems}

So far we have considered identification of a nominal model $G_{n}$ for an unknown system $G$. After identifying $G_{n}$ model quality estimation can be performed via validation/invalidation of uncertainty models.

An analogue to the model validation problems considered in $[15,14,19,4]$ is the following problem :

Does there exist a $\Delta G_{n} \in \mathcal{B}$ satisfying $\left\|\Delta G_{n}\right\| \leq$ $\rho(n, N)$ so that $y(t)=\left(\left[G_{n}+\Delta G_{n}\right] * u\right)(t)+\xi(t)$ for some $\xi(t), t=0,1, \ldots, N-1$, satisfying the error priors at hand?

Here $\rho(n, N) \geq 0$ is a given number. This model validation problem can be solved as an infinite linear programming feasibility problem if the error priors are linear as in section 4 ( $u$ is assumed to be known), and as a finite linear programming feasibility problem if $u(t)=0$ for $t<0$.

Various model validation problems for uncertain systems can be formulated as linear programming feasibility problems. One variation is to restrict the complexity of $\Delta G_{n}$ by setting $\Delta g_{n}(k)=0$ for $k>L$, where $L$ is some given positive integer.This results in a standard finite linear programming feasibility problem which can be solved efficiently with the method described in [1]. 


\section{Simulation Examples}

In this section we present computer simulations illustrating the application of the linear programming methodology to problems of modelling of uncertain systems.

The noise in the simulations has been generated using pseudorandom number generators, and typical sequences have been chosen.

Consider the system $G \in \mathcal{B}$ with transfer function

where

$$
\hat{G}(z)=\hat{H}(z) / \hat{E}(z),
$$

$$
\begin{gathered}
\hat{H}(z)=0.0387 z-0.0444 z^{2}-0.0461 z^{3}+ \\
0.0960 z^{4}-0.0153 z^{5}-0.0482 z^{6}+0.0260 z^{7} \\
\hat{E}(z)=1-4.7643 z+10.0325 z^{2}-11.8785 z^{3}+ \\
8.3276 z^{4}-3.2776 z^{5}+0.5671 z^{6}
\end{gathered}
$$

This corresponds to a sampled continuous system with a sampling time of $0.5 \mathrm{~s}$.

The system has three pairs of complex conjugate poles at $(0.8377 \pm 0.7012 i),(0.9259 \pm 0.4914 i)$ and $(1.1264 \pm 0.2758 i)$. The second pair of poles results in a poorly damped resonance at about $1.0 \mathrm{rad} / \mathrm{s}$, which dominates the impulse response of the system (Fig.2). The first and the third pair of poles result in more damped resonances at $1.4 \mathrm{rad} / \mathrm{s}$ and $0.3 \mathrm{rad} / \mathrm{s}$, respectively.

The simulated system is $y(t)=(G * u)(t)+v(t)$, where the noise $\{v(t)\}$ is generated as Gaussian white noise with variance 0.02 . In all simulations the input sequence $\{u(t)\}$ is generated as a PRBS (pseudorandom binary signal) sequence of the numbers \pm 1 with transitition period between 1 and 20 samplings.

\section{Identification of Nominal Model}

Because of the resonant property of the system we choose the Kautz model structure to correspond to the frequency and damping of the dominant resonance, which we assume to be known. For this example we simply place the poles of the Kautz mode at $(0.9259 \pm 0.4914 i)$

In order to model all three resonance peaks with a minimum number of parameters a combination of three Kautz models, each describing one resonance peak, would be needed [8]. In accordance with the assumption that only the dominating resonance is known, we select a model structure consisting of a Kautz model combined with a FIR model. The Kautz model should be able to accurately model the known resonance while the FIR-part of the model structure is used for modelling the more damped modes of the system. The Kautz model is selected to be of order 2 , the FIR model of order 20 .
The transfer function of the nominal model is thus

$$
\hat{G}_{n o m}(z)=\hat{K}_{n k}(z)+\hat{F}_{n f}(z),
$$

where $\hat{F}_{n f}(z)$ is a FIR model of order $n f$. The equivalent state space model is

$$
\begin{gathered}
\hat{x}(t+1)=A_{n} \hat{x}(t)+B_{n} u(t) \\
\hat{y}(t)=C_{n} T_{n} \hat{x}(t)
\end{gathered}
$$

where the elements of the matrices $A_{n}, B_{n}$ and $T_{n}$ for the Kautz part of the model are obtained from (8-10). For the case with $n k=2$, which is used below, the matrices are

$$
\begin{aligned}
& A_{n}=\left(\begin{array}{cccccccc}
b(1-c) & c & 0 & 0 & 0 & \ldots & 0 & 0 \\
1 & 0 & 0 & 0 & 0 & \ldots & 0 & 0 \\
0 & 0 & 0 & 0 & 0 & \ldots & 0 & 0 \\
0 & 0 & 1 & 0 & 0 & \ldots & 0 & 0 \\
0 & 0 & 0 & 1 & 0 & \ldots & 0 & 0 \\
\vdots & \vdots & \vdots & \vdots & \vdots & \ddots & \vdots & \vdots \\
0 & 0 & 0 & 0 & 0 & \ldots & 1 & 0
\end{array}\right) \\
& B_{n}=\left[\begin{array}{llllllll}
1 & 0 & 1 & 0 & 0 & \ldots & 0 & 0
\end{array}\right]^{\prime} \\
& T_{n}=\left(\begin{array}{ccccc}
\sqrt{1-c^{2}} & -b \sqrt{1-c^{2}} & 0 & \ldots & 0 \\
0 & \sqrt{\left(1-c^{2}\right)\left(1-b^{2}\right)} & 0 & \ldots & 0 \\
0 & 0 & 1 & \ldots & 0 \\
\vdots & \vdots & \vdots & \ddots & \vdots \\
0 & 0 & 0 & \ldots & 1
\end{array}\right)
\end{aligned}
$$

Here ' denotes vector transpose. The first two lines of $A_{n}, B_{n}$ and $T_{n}$ correspond to the Kautz-part of the model, the next $(n f)$ rows to the FIR-part of the model.

A data sequence of 800 input/output data pairs was generated. The first 200 inputs were used as input to the state space model (21) in order to let the state vector converge from an arbitrary initial state. The next 300 data pairs were used for identification and the last 300 data pairs for model validation.

The Bode diagram of the identified nominal model is in Fig. 1 compared to the Bode diagram of the system (17) and in Fig.2 the corresponding impulse responses are compared. It is seen that the dominating resonance at $1.0 \mathrm{rad} / \mathrm{s}$ is accurately modelled by the Kautz model, but that the 20th order FIR model cannot very accurately model the highfrequency response of the system. This inaccuracy of the identified nominal model is to be included in the model uncertainty $\Delta G_{n}$. The modelling error is $\left\|G-G_{n o m}\right\|=0.50$ and the value of the LSAD criterion, $R_{i d}=0.1066$.

\section{Model Validation}

We shall apply the model validation procedure outlined in section 5 . The feasibility of a complete 

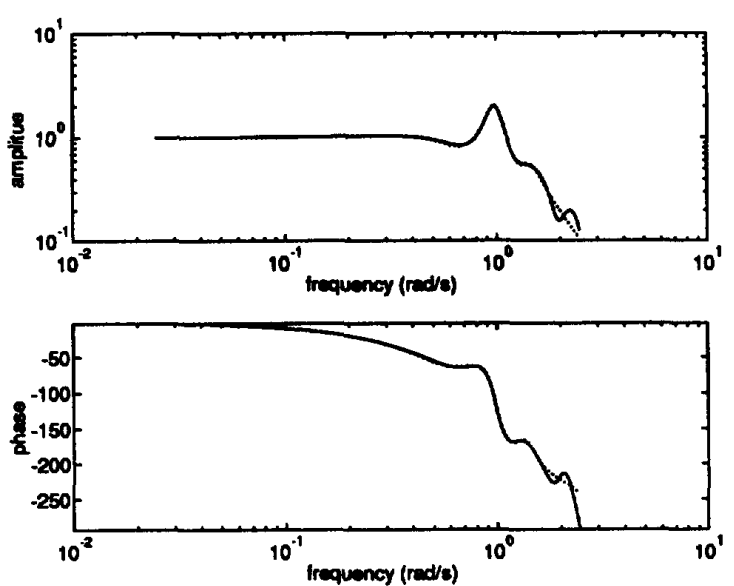

Figure 1: Bode diagrams of identified nominal model (continuous lines) and simulated system (dotted lines).

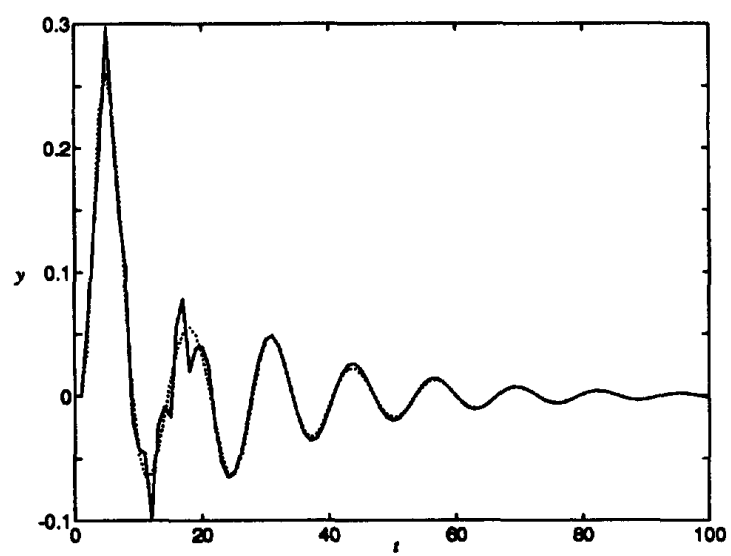

Figure 2: Impulse responses of identified nominal model (continuous line) and simulated system (dotted line).

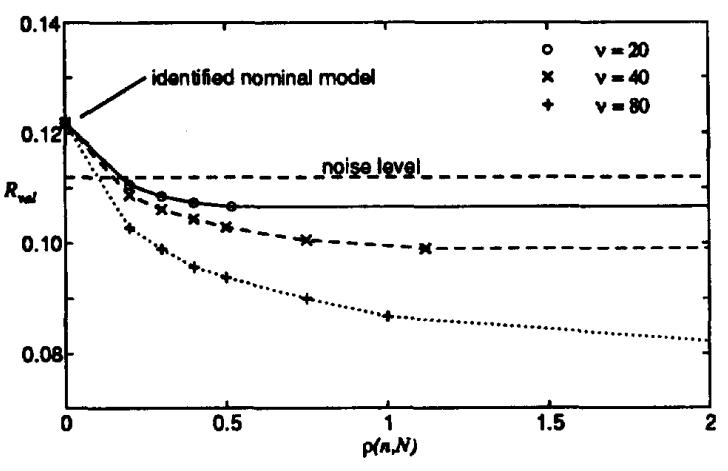

Figure 3: An example of model validation including uncertainty for three different uncertainty models.

uncertainty model $G_{\text {nom }}+\Delta G,\|\Delta G\| \leq \rho(n, N)$ $(\rho(n, N)>0$ is a given number), is determined by testing that

$y(t)=\left[\left(G_{n o m}+\Delta G\right) * u\right](t)+\xi(t), t \in$ validation set

for some $\Delta G$ satisfying $\|\Delta G\| \leq \rho(n, N)$, and for some bounded $\xi(t)$ such that $1 / N \sum_{t}|\xi(t)| \leq \epsilon$. Here the sum is taken over the validation set which is taken to consist of $N$ consequtive $t$-values. Furthermore, $\epsilon>0$ is some estimated bound for the average absolute value of the noise.

In the actual computations the perturbation $\Delta G$ is modelled as a FIR model of order $\nu$. The best estimate of the perturbation over the validation set, given the previously identified nominal model and given a constraint $\rho(n, N)$, is calculated with the LSAD method. The resulting value of the LSAD criterion, $R_{v a l}$, equals $1 / N \sum_{t}|\xi(t)|$.

Simulation results are shown in Fig. 3 for the three selections of uncertainty models, $\nu=20, \nu=40$ and $\nu=80$, and for a few values of $\rho(n, N)$.

If $\epsilon$ is taken to be 0.11 , which is the actual average absolute noise level, the smallest unfalsified size for the perturbation is between 0.07 to 0.15 depending on the complexity of the perturbation model. These values are far too small compared to the actual value of $\left\|G-G_{\text {nom }}\right\|=0.50$ and thus the smallest unfalsified perturbation should not be used as the size of the unmodelled dynamics.

A better estimate is obtained through minimization of $1 / N \sum_{t}|\xi(t)|$ without the constraint on $\|\Delta G\|$. Care must, however, be taken not to select a too complicated model for the perturbation, which will lead to overfitting to noise and to too conservative values for the size of $\triangle G$. This method gives the sizes 0.52 and 1.12 for $\nu=20$ and $\nu=40$, respectively. A perturbation model order of $\nu=80$ is clearly too complex and 
would give a size of the perturbation greater than 2 .

Acknowledgement Financial support from the Academy of Finland to the authors is gratefully acknowledged.

\section{REFERENCES}

[1] Barrodale, I. and F.D.K. Roberts (1978). An efficient algorithm for discrete $l^{1}$ linear approximation with linear constraints. SIAM J. Numer. Anal., 15, 603-611.

[2] Dahleh, Mohammed and R. Smith (eds) (1993). Proc. Workshop Uncertainty Modeling in Control Systems. Springer. In print.

[3] Dahleh, M.A., T. Theodosopoulos and J.N. Tsitsiklis (1993). The sample complexity of worstcase identification of F.I.R. linear systems. Systems and Control Letters, 20, 157-166.

[4] Gustafsson, T.K. and P.M. Mäkilä (1993). Modelling of uncertain systems via linear programming. Submitted. A shortened version presented at the 1993 IFAC World Congress, Sydney.

[5] Helmicki, A.J., C.A. Jacobson and C.N. Nett (1991). Control-oriented system identification: A worst-case/deterministic approach in $H^{\infty}$. IEEE Trans. Automat. Control, 36, 1163-1176.

[6] Kautz, W.H. (1954). Transient synthesis in the time domain. I.R.E. Trans. Circuit Theory, CT1(3), 29-39.

[7] Kosut, R.L., G.C. Goodwin and M.P. Polis, Guest Editors. (1992). Special Issue on System Identification for Robust Control Design. IEEE Trans. Automat. Control, 37, 899-1008.

[8] Lindskog, P. and B. Wahlberg (1993). Applications of Kautz Models in System Identification. Proc. 12th IFAC World Conf., Sydney.

[9] Mäkilä, P.M. and J.R. Partington (1992). Worstcase identification from closed-loop time series. Proc. 1992 American Control Conf., 301-306.

[10] Mäkilä, P.M. and J.R. Partington (1993). On bounded error identification of feedback systems. International J. Adaptive Control and Signal Processing. To Appear.

[11] Milanese, M. and A. Vicino (1991). Optimal estimation theory for dynamic systems with set membership uncertainty: An overview. Automatica, 27, 997-1009.

[12] Narula, S.C. and J.F. Wellington (1979). Selection of variables in linear regression using the minimum sum of weighted absolute errors criterion. Technometrics, 21, 299-306.

[13] Partington, J.R. and P.M. Mäkilä (1993). Worst case analysis of time domain identification methods. Submitted.

[14] Poolla, K., P. Khargonekar, A. Tikku, J. Krause and K. Nagpal (1992). A time-domain approach to model validation. Proc. 1992 American Control Conf., Chicago.

[15] Smith, R.S. and J.C. Doyle (1992). Model validation: A connection between robust control and identification. IEEE Trans. Automat. Control, 37, 942-952.

[16] Söderström, T. and P. Stoica (1991). System Identification. Prentice-Hall, Englewood Cliffs.

[17] Wahlberg, B. (1991). System identification using Laguerre models. IEEE Trans. Automat. Control, 36, 551-562.

[18] Wahlberg, B. (1991). Identification of resonant systems using Kautz filters. Proc. 30th IEEE Conf. Dec. Control, Brighton.

[19] Zhou, T. and H. Kimura (1992). Identification for robust control in time domain. Preprint. 